\title{
Relaxing Global-As-View in mediated data integration from Linked Data
}

\author{
Alessandro Adamou \\ Data Science Institute, Insight Centre, NUI Galway \\ Lower Dangan, Galway, (Ireland) \\ alessandro.adamou@nuigalway.ie
}

\author{
Mathieu d'Aquin \\ Data Science Institute, Insight Centre, NUI Galway \\ mathieu.daquin@nuigalway.ie
}

\begin{abstract}
In scenarios where many different, independent and dynamic data sources need to be brought together, mediated data integration at runtime is rapidly gaining interest. In a global-as-view approach, schema mappings express how to get data from each data source according to the global schema of the mediator. Key issues include the effort required to include and map new data sources, and the very need of data sources for the global schema to be expressed. It has been argued that the principles of Linked Data can be used to spread the cost of adding new sources in a pay-as-you-go model. We contribute by describing a data integration framework able to mitigate these issues, by relating data sources under a global schema which is implicit and only partly known at the time a new data source joins. Mappings over a data source only require partial knowledge of it and of the part of the global schema that it will affect. Pay-as-you go can then be employed to guarantee eventual schema compliance. This approach was adopted in a large-scale data integration system for Smart Cities, where it allowed short time-to-publish for new data and iterative schema refinements.
\end{abstract}

\section{CCS CONCEPTS}

- Information systems $\rightarrow$ Mediators and data integration; Semantic web description languages; Data centers.

\section{KEYWORDS}

View-based data integration, Linked Data, Smart Cities

\section{ACM Reference Format:}

Alessandro Adamou and Mathieu d'Aquin. 2020. Relaxing Global-As-View in mediated data integration from Linked Data. In Semantic Big Data (SBD'20), fune 14-19, 2020, Portland, OR, USA. ACM, New York, NY, USA, 6 pages. https://doi.org/10.1145/3391274.3393635

\section{INTRODUCTION}

One modern use case of data integration is to make data coming from disparate sources available to consumers, such as developers, through a unified endpoint, more commonly an HTTP-based Web Service API. These services present a view over the data according to a (global) schema and are usually backed by one of two strategies. In materialized data integration (or data warehousing), the data are

Permission to make digital or hard copies of part or all of this work for personal or classroom use is granted without fee provided that copies are not made or distributed for profit or commercial advantage and that copies bear this notice and the full citation on the first page. Copyrights for third-party components of this work must be honored

For all other uses, contact the owner/author(s).

SBD'20, June 14-19, 2020, Portland, OR, USA

(C) 2020 Copyright held by the owner/author(s).

ACM ISBN 978-1-4503-7974-8/20/06.

https://doi.org/10.1145/3391274.3393635 copied onto one site in an extract-transform-load (ETL) process. In mediated (or virtual) data integration, the data to be integrated still reside on the original sources, only to be fetched and transformed as the need arises [3], such as when the API receives a request and needs to query the original data sources, or a subset thereof.

The lazy approach of virtual data integration has been gaining interest from the industry [12], because it suits the economy of the small and medium enterprise that cannot afford the capacity required by data warehousing, but can instead commit some computational or network resources. It also offers a way to keep the integrated data up to date. Note that in virtual integration, more than in other types of integration, not only can the schemas of the data on their sources differ, but also the languages for querying them can differ from one another, and from the query language at the endpoint (e.g. the syntax and semantics of URL construction).

From the literature, there are two possible ways of performing view-based data integration. In local-as-view (LAV), the mappings associate a query over the global schema to each element of the source schema; in global-as-view (GAV), they associate a query over the source schema to each element of the global schema [17]. A third category of mapping languages, GLAV, generalizes both [5].

$\mathrm{GAV}$ is known for its general modelling power when designing the global schema, and for its ease of query processing (for instance by executing procedural mappings), if at a greater cost of adding a new source than in LAV. If a new data source needs to be integrated, it may contain data not contemplated in the model of the global schema, thereby requiring a re-definition of some elements of the global schema and of the mappings associated with them. There is also a potential fault tolerance problem: a source schema suddenly changing may cause either all of its data to be rejected by the integration system, or the integration system to fail altogether. At the core of these issues is the need for data sources to exist, and for a global schema to be known in its entirety at the time of mapping definition, so that GAV mappings can be written for all its symbols.

The Linked Data (LD) paradigm deals with the identifiability of entities in the data and the ways in which they reference one another using links with a shared meaning. It also dictates that the information about the entities should be exchanged according to globally understood conventions and protocols. In the reference LD technology stack, standards include URIs, the RDF format(s) and the SPARQL query language. Other characteristics are monotonicity (adding new facts does not cause the removal of existing ones from a knowledge base) and the open world assumption (a fact not present in the knowledge base could still be true).

In this paper, we want to address the question: How can Linked Data principles be adopted in a mediated GAV data integration system in order to mitigate, or at least defer, some of its known issues? 
We will illustrate some ways in which the characteristics of LD can be exploited to: (i) reduce the need to comply with a fully defined apriori global schema; (ii) incrementally refine the integration rules starting from an easy initialization phase. We choose GAV as a preferred way of formulating the integration rules, then relax the constraints for global schema compliance while maintaining those on the schemas of the local data sources. Part of the approach is the possibility to define integration rules not as full queries over the local schemas, but as reusable primitives which, when combined, generate such queries. These primitives are organized in structures that reflect those of knowledge organization systems like ontologies and thesauri, and can be defined globally or on a per-dataset basis.

While our approach does not completely remove the need for redesigning the global schema when a new data source is added, it allows the process to be controlled locally. We offer an insight as to how to use LD principles to obtain a data integration system that:

(1) is mediated (virtual);

(2) uses mapping rules that are similar to GAV ones, but

(3) is schema-later: it does not need the global schema to be fully defined, but can mitigate the side effects of this;

(4) is pay-as-you go: the mappings can be refined incrementally based on feedback, or adapted to new data sources.

An implementation of the underlying principles of this framework exists in the form of a data API for a Smart Cities project for the city of Milton Keynes, UK, owing to the fact that Smart Cities are a domain where it is very hard for a global schema to be designed a priori by a single data engineering unit. It was therefore deemed a suitable setting for use cases that justify this framework.

\section{SETTING}

In what follows, we assume to have a HTTP API that uses a conjunctive query language, whose answers are full descriptions of all the matching entities in the data, e.g. "Tell me everything you know about local areas with postcode MK6". ${ }^{1}$ The API is backed by a view-based data integration system (VDIS) which, upon receipt of a query, aggregates the answers from corresponding queries over several datasets. These datasets follow LD principles, in that their representation formats are standardised and their local schemas share some terms, such as entity types and properties.

The manager of the integration endpoint is responsible for designing the global schema of the data returned by the API. New data sources are registered independently, whereupon the integration manager writes the rules that map it to the global schema. While local data providers do not write mappings for their datasets, they may offer feedback on how their data appear in the integrated view.

As the integrated view also follows LD principles, implying monotonicity and open world, no claim to completeness is made over the global schema. While one does exist, it is not fully explicit: only the symbols for which some rule has been written are explicit. As we will see, though, not all these rules need to be dataset-specific.

We are in an ideal scenario for GAV mappings, where the global schema is expressed in function of the local ones. We therefore make another important assumption: for every element of the global schema, a query over a local schema can be written in isolation. In

\footnotetext{
${ }^{1}$ This is a common paradigm in RESTful Web Services, where every query returns a description of the state of a resource, and as such it is a sensible scenario.
}

other words, the mappings do not depend on other queries being answered by other datasets first. For example, a global view will not need a dataset to answer a query that identifies schools in an area before it can query another dataset for the bus stops near those schools. Indeed, cross-dataset mappings are not part of the GAV canon because mappings target one source schema at a time, though they may be included in hybrid or derivative systems [5].

In the following, we will show how, under these realistic conditions, it is possible to avoid (or at least delay) redesigning the global schema to accommodate a new data source joining the VDIS.

\section{RELATED WORK}

We have already introduced the key notions of materialized vs. mediated, as well as view-based data integration, from the literature $[3,5,17]$. Recent methods, emerging from the world of Big Data, advocate the adoption of laziness and incrementality in data integration. The pay-as-you-go (PAYG) concept embraces both: PAYG techniques aim at consuming as little upfront effort as possible, to instead spend additional work as the system is interacted with [20].

With the emergence of Linked Data, Paton et al elaborated on its potential for the realization of PAYG integration systems. They argued that PAYG can be combined with Linked Data to improve data structure sharing, entity linking and query rewriting to different structures [19]. This is one of the main inspirations of our work, which aimed at modelling a system that realized its vision.

Approaches also differ on the way they view data schemas, particularly the global schema, and enforce compliance with them. Lazy approaches attract research interest on that front as well: Berzal et al theorized on the possibility of using "lazy types", i.e. data types whose structure and behavior dynamically adapt to the available data [4]. Their use of an object-oriented language to illustrate this approach hinted at both the procedural nature of mappings and the hierarchical models typically found in ontologies. Notions like schemalessness were later introduced to denote that data integration systems should tolerate mapping rules that do not strictly adhere to the global schema. Schema-later combines this with laziness, to denote that the schema is refined as more details of the data sources become known. This leads to schema evolution, which is addressed for instance in PRISM, a system specifically designed for the use case of database migration [7].

Ontologies are the main means of building schemas for linked datasets, thus several ontology-based data integration systems exist $[6,16]$. Their focus is entity linking, as they rely upon leveraging existing instance-level equivalence. More broadly, there have been several applications of Linked Data to data integration. Strobin et al tackled the case of building entity summaries by following links across multiple datasets [21]. There are also LAV-based semantic data integration systems, such as the one by Martín et al [18], which focussed on the cross-referencing problem, and Karma [15]. The latter allows users to design the global schema as an ontology and to write transformation scripts from tabular and hierarchical data. While they demonstrate the effectiveness of Linked Data in LAVbased integration, they call for a reflection on how the GAV model can be adapted to fit similar contexts. Dimartino et al argue [10] that a single ontological conceptualization is not feasible in semantic integration, which requires an extensible approach instead. 


\section{INTEGRATION FRAMEWORK}

Let $\mathcal{I}=(\mathcal{G}, \mathcal{S}, \mathcal{M})$ be a virtual VDIS with global schema $\mathcal{G}$ and $n$ data sources with schemas $\mathcal{S}_{1} \ldots \mathcal{S}_{n}$ respectively (overall constituting $\mathcal{S}$ ). $\mathcal{M}$ is the mapping specification that must be designed for $\mathcal{I}$. If $\mathcal{M}$ is GAV, then it assigns a query $q_{\mathcal{S}_{i}}$ over $\mathcal{S}_{i}$ to each single element $g$ of $\mathcal{G}$ [17]. Mappings will therefore be of the form

$$
g \leadsto q_{S_{i}}
$$

Operationally speaking, the integration logic will inspect each symbol of $\mathcal{G}$ and attempt to execute all the mappings with that symbol (the only one that appears) in the body, hence the "procedural" flavor of GAV mentioned in the introduction. We defer for now any details on what the $q_{S_{i}}$ themselves look like.

We shall now relax the assumption that $\mathcal{G}$ is entirely given and make it emerge from the iterative design of schema mappings. Without loss of generality, we can assume the ordering of the local schemas $\mathcal{S}_{i}$ reflects the order in which they join the VDIS. To assume that $\mathcal{G}$ is not known means we only have knowledge of some of its symbols at a given time. This is treated like having a different global schema at that time, with only those symbols in it.

Definition 1. Given a source schema $\mathcal{S}_{i}$, let $\mathcal{G}_{j}, j \geq i$ be a schema for which GAV mappings between $\mathcal{G}_{j}$ and $\mathcal{S}_{i}$ exist and so that:

(1) $\mathcal{G}_{1}$ is not empty

(2) if $\mathcal{R}_{i}, i>1$ is the set of relational symbols of $\mathcal{G}_{i}, \mathcal{R}_{i-1} \subseteq \mathcal{R}_{i}$ We call $\mathcal{G}_{j}$ an intermediate schema for $\mathcal{I}$ with respect to $\mathcal{S}_{i}$.

In other words, an intermediate schema contains the relations and attributes that were given to the owner of a data source at the time the integration rules for that source had to be designed.

\subsection{Relaxation in schema mappings}

We denote with $R[\mathcal{S}]$ the set of relation symbols of a schema $\mathcal{S}$, and with $a[\mathcal{S}]$ the set of attribute symbols of $\mathcal{S} .^{2}$

Definition 2. An intermediate schema mapping $\mathcal{M}_{i j}$ for a source schema $\mathcal{S}_{i}$ and schema $\mathcal{G}_{j}$ is a set of rules of the form

$$
\left(g,\left\{a_{l}\right\}\right) \leadsto q_{\mathcal{S}_{i}}
$$

where $g$ is a relation symbol of $\mathcal{G}_{j}$, the $\left\{a_{l}\right\}$ are attributes in $a\left[\mathcal{G}_{j}\right] \cup$ $a\left[\mathcal{S}_{i}\right]$ and the $q_{\mathcal{S}_{i}}$ are queries over $\mathcal{S}_{i}$.

Unlike standard GAV, we specify the attributes $a_{l}$ in the head because they are not given: only those from $a\left[\mathcal{G}_{j}\right]$ are in the specification of the data integration system. If the $a$ are all in $a\left[\mathcal{G}_{i}\right]$ then an intermediate mapping is like a classical GAV mapping but on a part of the global schema; if there are attributes from $a\left[\mathcal{S}_{i}\right]$ it means that the integration manager is 'proposing' the addition of new properties to the global schema. This extension to local schema attributes is important, because it allows to define mappings that bring in new attributes into the global schema, by either transforming them and their values, or keeping them as they are.

Given $\mathcal{G}_{j}$ and the new local schema $\mathcal{S}_{i}$, the integration manager produces a rule of the form $\left(g,\left\{a_{l}\right\}^{g}\right) \leadsto q_{\mathcal{S}_{i}}$ for every $g \in \mathcal{G}_{j}$. For each $g$, we may therefore have one of the following:

${ }^{2} \mathrm{~N}$-ary relations can be built in Linked Data through combining the binary relations of RDF triples in reification, and single RDF properties can be treated as attributes.
(1) if $a_{g}=a\left[\mathcal{G}_{j}\right]$, the mapping is equivalent to one of the form $g \leadsto q_{\mathcal{S}_{i}}$, i.e. a normal GAV mapping, because it is returning the expected attributes for $g$ in $\mathcal{G}_{j}$.

(2) if $a_{g} \subset a\left[\mathcal{G}_{j}\right]$, the mapping is associated to a relation of lower arity than the one of that relation in $\mathcal{G}_{j}$.

(3) if $a\left[\mathcal{G}_{j}\right] \subset a_{g}$, the mapping is 'proposing' that some attribute of $\mathcal{S}_{i}$ not in $\mathcal{G}_{j}$ be considered for addition to $\mathcal{G}_{j}$.

(4) $a_{g}$ and $a\left[\mathcal{G}_{j}\right]$ are neither equal nor subsets of one another.

To restrict to the GAV canon, where a query is assigned to every symbol of $\mathcal{G}_{j}$, we should rule out cases 2 and 4 . Even then, however, we will show that $\mathcal{G}_{j}$ can still be fully covered by the mapping. As part of cases 3 and 4, we now introduce an operator that pulls attributes from the local schema and preserves them.

If $\mathcal{M}_{i j}$ is the set of mappings over $\mathcal{G}_{j}$ and $a\left[\mathcal{M}_{i j}\right]$ are the attributes that appear in the heads of all the mappings of $\mathcal{M}_{i j}$, then:

Definition 3. A mapping of the form $(g, *) \sim q_{\mathcal{S}_{i}}$ is such that $q_{\mathcal{S}_{i}}$ is the identity over all the attributes $a\left[\mathcal{S}_{i}\right] \backslash a\left[\mathcal{M}_{i j}\right]$.

Executing a mapping with the star operator brings in all the attributes from the local data source that are not dealt with by any other intermediate mapping, as in a SELECT * in SPARQL when there is a property variable. We justify the presence of such a kind of mapping on our assumption of working with Linked Data.

(1) By monotonicity, new data do not invalidate existing ones: we can then tolerate the addition of new attributes to $\mathcal{G}_{j}$.

(2) since LD mandates reuse of common vocabularies [13], there is a likelihood that new attributes could be shared across local schemas and even globally understood if they are standards.

We began by defining mappings from explicit parts of the global schema, then added a way to directly bring in elements from a local schema. The outcome of it might be imperfect and require to be refined, based e.g. on feedback from the data provider or the affected applications. This can be represented by a function $\Phi^{\mathcal{G}}(j, k)$ that quantifies the "price paid" by transitioning from $\mathcal{G}_{j}$ to $\mathcal{G}_{j+k}$.

GAV expresses the global schema in function of the local ones, which means that some data source is needed before any part of the global schema can be defined. As part of the Linked Data principles is the reuse of common properties, there is value in trying to define, or refine, part of the global schema based on these standard vocabularies, even in the absence of data sources. A way to formalize this is by treating intermediate schemas as if having the same capacity of having mappings written over as local schemas.

Definition 4. An endomapping over a global schema $\mathcal{G}$ is a mapping between $\mathcal{G}_{j}$ and $\mathcal{G}_{j+1}$.

Instead of the source schema, endomappings associate queries over a certain version of the global schema to elements of the next version of the global schema. They are basically rewrite rules for the global schema onto itself, yet at different stages of it: for example, to refine an attribute newly added by a mapping that uses a star operator, in response to feedback in the pay-as-you-go model.

Example. Suppose local schema $\mathcal{S}_{1}$ has the following relation: demographic(district_id,name,area,pop_2008,pop_2009) and we need a mapping from the intermediate global schema $\mathcal{G}_{j}$ : district(district_id,name) 
which has no attributes for historical data about population. Intending to allow for one, we create mappings over another relation, district*, which incorporates the attributes from demographic:

district*(district_id, name, area,pop_2008, pop_2009)

$$
\leadsto\{x, y, z, v, w \mid \text { demographic }(x, y, z, v, w)\}
$$

Since having one attribute per year of observation is less than ideal, this can be rectified either by refining the revising the mappings over $\mathcal{S}_{1}$, or by rewriting the data internally with an endomapping. Also, area gets rewritten into surface to avoid confusion with local areas. The refined global schema $\mathcal{G}_{j+1}$ looks like this:

\section{district(district_id,name,surface)}

population(district_id,year, value)

This can be done by using the following endomapping:

$$
\begin{gathered}
\text { district(district_id,name,surface }\}) \\
\sim\{x, y, z \mid \text { district } *(x, y, z, v, w)\} \\
\text { population(district_id, }{ }^{\prime} 2008^{\prime}, \text { value) } \\
\leadsto\{x, v \mid \text { district } *(x, y, z, v, w)\} \\
\text { population(district_id, }{ }^{\prime} 2009^{\prime}, \text { value) } \\
\leadsto\{x, w \mid \text { district } *(x, y, z, v, w)\}
\end{gathered}
$$

In further refinements, a procedural mapping can capture the year value by parsing the population_\{year $\}$ attribute names.

It has been mentioned that conforming to the Linked Data principle of vocabulary reuse entails a likelihood of reducing the price paid as the global schema emerges. By a similar line of reasoning, it makes sense to try and reuse the elements of a query across local data sources that share that kind of similarity. The following section illustrates a way of structuring fragments of a query for reuse.

\subsection{Reusable query elements}

Recall that an element of a GAV-like mapping produces a query $q_{S_{i}}$ over the local schema from a symbol of the global schema. It is possible to make the generation of the queries themselves parametric, by taking advantage of the semantics of the global schema, and to do so at runtime, even at the time of rewriting the global schema query in one language into a local schema query in another language. We therefore call this process fust-in-time (IIT) query recompilation, described in a previously published paper [1], which this section summarizes with additional examples.

We define two types of primitives used for dynamically assembling a query: microcompiler and query skeleton.

A microcompiler is a function $\phi: \mathbb{W} \rightarrow \Sigma^{*}$ that transforms attribute-value pairs $(a, v) \in \mathbb{W}$ into a sequence of symbols of $\Sigma$. For example, given the a-v pairs $\{($ name, portland),(state, or $)\}$, a basic microcompiler takes their conjunction and produces the DBpedia resource dbr: Portland,_OR through simple syntactic heuristics.

Unlike standard GAV mappings, a microcompiler determines the association not of a query, but of a query element $-\Sigma^{*}$ in this example is the language of SPARQL nodes, which URIs are part of - to one or more symbols of the global schema (name and state).

A query skeleton, or query template, $t$ is a member of $(\Sigma U$ $C)^{*}$, where $C$ is an alphabet of control symbols: it extends the target language with such symbols. In the example, if $\Sigma^{*}$ is now the language of triple patterns in SPARQL, then the following: ?[ctrl_ox]? (dbo: hometown $\mid$ dbo: residence) <[place]>

is a query skeleton that produces a triple pattern to find anyone (or anything) that either lives or hails from somewhere. Here, ? [ctrl_ox]? and $<[$ place $]>$ are control symbols of two different types: the former is a placeholder for a SPARQL variable (the people we want to find) to be assigned at runtime. The latter is a placeholder for a SPARQL node, which in turn could become anything at runtime: a URI, a literal or even a set of more triple patterns (this is not known at the time of defining the query skeleton). ${ }^{3}$

For another dataset - or, in fact, many other datasets due to the standard nature of the vocabulary used - an applicable query skeleton for the from predicate could be:

\section{?[ctrl_ox]? foaf:based_near <[place]>}

Supposing the above primitives are assigned explicitly to the DBpedia dataset, then a combination of the above with SPARQL as the target language produces the following query: ${ }^{4}$

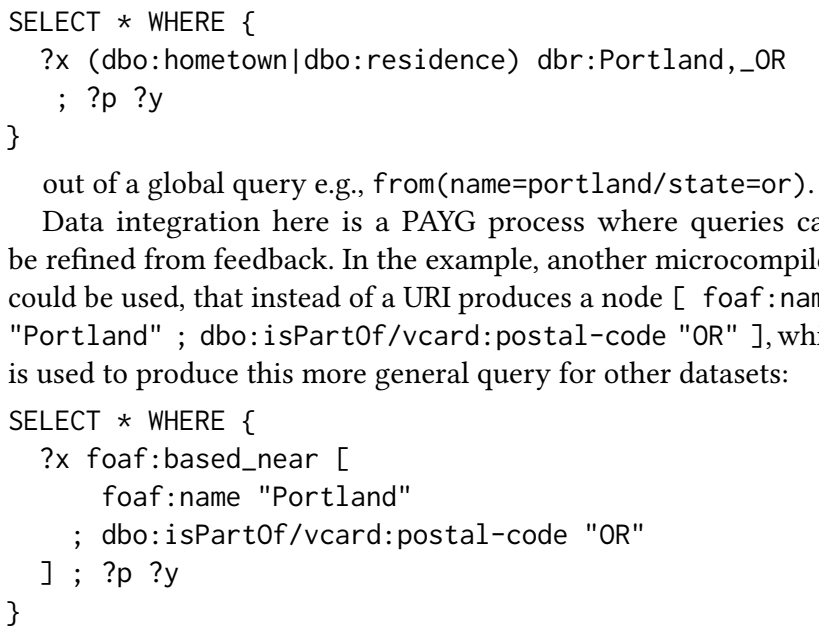
is used to produce this more general query for other datasets:

Data integration here is a PAYG process where queries can be refined from feedback. In the example, another microcompiler could be used, that instead of a URI produces a node [ foaf: name "Portland" ; dbo: isPart0f/vcard:postal-code "OR" ], which

A microcompiler declares what attributes it supports: either one of the above examples supports name and state (in the query language over the global schema) and acts upon their values differently: both will convert the name value to title case and make state uppercase, but have different ways of further manipulating them. How they do so is up to who defines the integration rules, but as this example showed, they can be incrementally refined as their knowledge of the target dataset evolves.

There needs to be a strategy in place, in order to select which microcompilers to evaluate and which query skeletons to combine them with. These depend on what we decide to attach the primitives to: the above queries are examples of intermediate mappings with a star operator assigned to more than one global symbol. However, they can even be tailored to a specific dataset, whereupon they override any other primitives defined for the same attributes but irrespective of the target dataset. One possible strategy, which works for knowledge graphs, where entities are commonly organised taxonomically, is to assign query skeletons to entity types, that is, to a-v pairs where the attribute is type and the value, e.g. place, is made explicit. An algorithm that cascades the selection of

\footnotetext{
${ }^{3}$ The types and meanings of control symbols are not prescriptive: the one shown is but one possible configuration.

${ }^{4}$ For brevity, we replace URIs with qualified names.
} 
primitives based on entity type and dataset, and that considers the class hierarchy of entity types, can be found in [1]. The paper also presents the evaluation of the method in terms of its ability to transform a query federation system into a mediated data integration system, whilst keeping the overhead manageable.

\section{APPLICATION TO URBAN OPEN DATA}

Smart Cities are a dynamic and data-intensive application domain, being very susceptible to changes in its use cases alongside the needs and habits of citizens. A data integration system servicing a smart city has to accommodate such dynamism: as more local authorities and other urban data providers are engaged, they are likely to offer datasets that cover subject areas - such as water or energy consumption - not encompassed by the global schema until then. For that reason, it is also difficult to design a global schema.

Not only should the addition of new sources with new local schemas not cause faults in existing data integration, but it could make sense for the new data to be included quickly in the integrated view, possibly even before refactoring the global schema. The early inclusion of these new data in some form is likely to generate feedback - a form of "price paid" in a PAYG system - which can be used to refine the global schema to accommodate them.

The case of Milton Keynes, UK, is one such occurrence. Its smart city undertakings were projects and initiatives funded throughout the 2010s: first MK:Smart ${ }^{5}$, then cityLABS ${ }^{6}$. Their policy was to gather data from as many sources as possible, and to offer them to the open data market early [9]. Datasets and service endpoints were catalogued in a single online resource: the MK DataHub ${ }^{7}$, which comprises a dataset registry, distributed storage solutions, and HTTP APIs for delivering streaming and static data [8] (Fig. 1).

An entity-centric API offers a unified access point to all the nonstreamed, distributed data delivered by providers registered with the MK DataHub. Like with many RESTful APIs, the query language is encoded in the URI scheme of the API itself. For example:

\{host\}/local_area/postcode/MK6

corresponds to a query to describe any entity of type local_area whose postcode is MK6. Descriptions are aggregated from datasets that satisfy this query locally, with their own schemas and query languages. To obtain them, the API is backed by a mediated VDIS.

Many registered datasets are statistics provided by several local authorities, such as the Milton Keynes Council, as well as by UKwide open data providers like the Land Registry ${ }^{8}$ and Ordnance Survey. ${ }^{9}$ Most local authorities offer their data as spreadsheets exported to CSV, each sheet representing an observation. To make them queriable, they are mirrored onto triple stores with SPARQL endpoints, however the onboarding process does not substantially alter the data and preserves the schema of statistical observations. For example, the following snippet in simplified RDF/Turtle syntax:

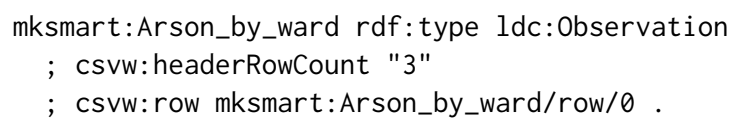

${ }^{5} \mathrm{MK}:$ Smart: https://www.mksmart.org/

${ }^{6}$ cityLABS: https://www.citylabs.org.uk/

${ }^{7}$ MK DataHub: https://datahub.mksmart.org

${ }^{8} \mathrm{HM}$ Land Registry Open Data, https://landregistry.data.gov.uk/

${ }^{9}$ Ordnance Survey open data, https://www.ordnancesurvey.co.uk/business government/tools-support/open-data-support

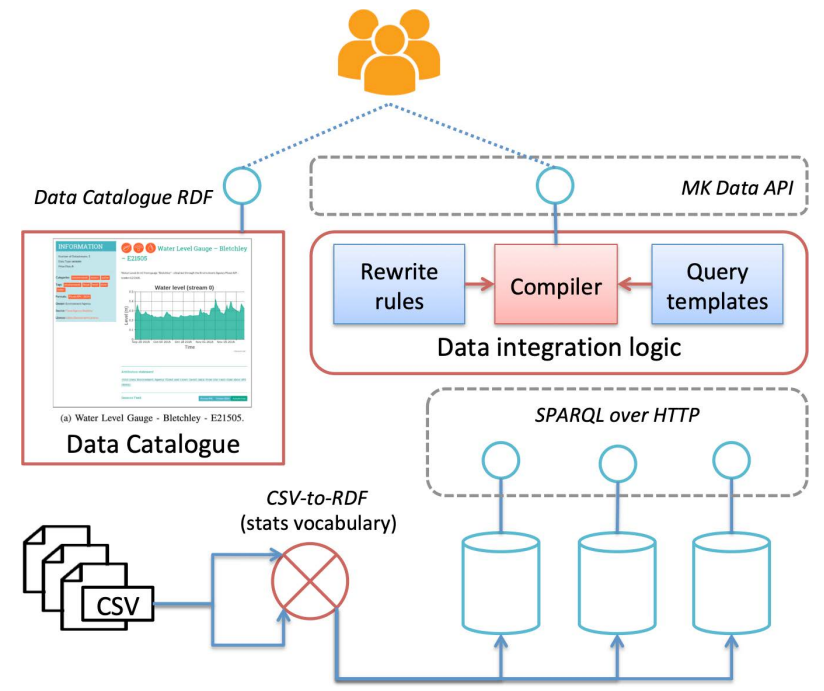

Figure 1: Pipeline of statistical data in the MK Data Hub, from CSV files to integrated APIs.

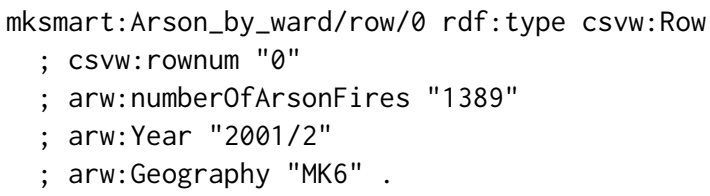

represents a statistical observation which has several rows, and one of them (row 0) says that there were 1389 arson fires during the period called "2001/2" in the area with code MK6. The local schema combines ad-hoc terms (arw) with standard vocabularies like Data Cube $(\mathrm{ldc})^{10}$ and $\mathrm{CSVW}^{11}$ to represent statistical and tabular data. This allows the VDIS to reuse query elements across several statistical datasets, but rewriting attributes and values so that the result published by the entity-centric API looks like this:

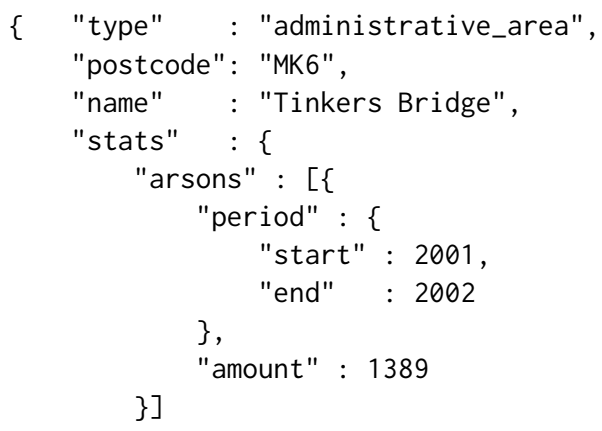

\})

The reference implementation of this data integration architecture, which was included with the MK DataHub, is available as open source. ${ }^{12}$ Mappings are written imperatively as simple JavaScript functions, owing to the procedural flavor of GAV [14]. Their application is cascaded following (i) the presence of dataset-specific mappings, and (ii) the ontological hierarchy of entity types.

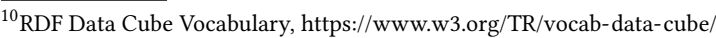

${ }^{11} \mathrm{CSV}$ on the Web, https://www.w3.org/ns/csvw\#

${ }^{12}$ Entity-Centric API, https://github.com/mk-smart/entity-centric-api
} 


\section{CONCLUSION}

Thanks to its standardization, Linked Data is an enabler for pay-asyou-go data integration. We have therefore presented a mediated integration framework inspired by GAV, which leverages the characteristics of Linked Data to partly overcome crucial drawbacks of GAV. Under realistic assumptions, it overcomes the inability to "model any information not present in at least one source" [14], because there is no longer a need for each global attribute to be explicitly specified from the start, and local attributes could be accepted as global until rewritten. Another known issue is that, although a data source can be registered with the VDIS independently, one still has to inspect whether its addition affects existing mappings, and rewrite them as necessary: while our approach does not solve the issue, it mitigates it by making the system schema-later and tolerant to importing local schema elements.

While it could be argued that our approach is closer to GLAV, we regard it as a relaxation of GAV, because when using intermediate mappings with the star operator, local attributes are not explicit in the mappings - unlike required by the LAV portion of GLAV - until incorporated in the global schema. Also, a LAV approach would fall short of practicability in such an environment, as it would require a much greater effort to monitor the evolution of the global schema to counter the potential obsolescence risk of every local mapping.

An unexplored limitation of the approach is how to address the cross-dataset problem when there is a dependency between data sources, i.e. the results of one query are necessary before one to another dataset may be executed. In particular, we will be exploring the applicability of recursive query planning [11] to this context.

There are classical challenges in data integration, such as inconsistencies in the formats and structures of the representation, which Linked Data alone is not meant to solve. However, we believe that this paradigm will help making these issues manageable while realizing data integration systems progressively more flexible.

\section{ACKNOWLEDGMENTS}

This work was supported by the projects MK:Smart (HEFCE) and AFEL (EU H2020, grant agreement No. 687916), and by the Insight SFI Research Centre for Data Analytics (grant SFI/12/RC/2289_P2).

\section{REFERENCES}

[1] Alessandro Adamou, Mathieu d'Aquin, Carlo Allocca, and Enrico Motta. 2017 Supporting virtual integration of Linked Data with just-in-time query recompilation. In Proceedings of the 13th International Conference on Semantic Systems, SEMANTICS 2017, Amsterdam, The Netherlands, September 11-14, 2017, Rinke Hoekstra, Catherine Faron-Zucker, Tassilo Pellegrini, and Victor de Boer (Eds.) ACM, 112-119. https://doi.org/10.1145/3132218.3132227

[2] Marcelo Arenas, Óscar Corcho, Elena Simperl, Markus Strohmaier, Mathieu d'Aquin, Kavitha Srinivas, Paul T. Groth, Michel Dumontier, Jeff Heflin, Krishnaprasad Thirunarayan, and Steffen Staab (Eds.). 2015. The Semantic Web - ISWC 2015 - 14th International Semantic Web Conference, Proceedings, Part I. LNCS, Vol. 9366. Springer. https://doi.org/10.1007/978-3-319-25007-6

[3] Leopoldo E. Bertossi and Loreto Bravo. 2005. Consistent Query Answers in Virtual Data Integration Systems. In Inconsistency Tolerance [result from a Dagstuhl seminar], Leopoldo E. Bertossi, Anthony Hunter, and Torsten Schaub (Eds.), Vol. 3300. Springer, 42-83. https://doi.org/10.1007/978-3-540-30597-2_3

[4] Fernando Berzal Galiano, Juan Carlos Cubero Talavera, Nicolás Marín, and María Amparo Vila Miranda. 2006. Data Integration Using Lazy Types. In Advances in Hybrid Information Technology, First International Conference, ICHIT 2006, Jeju Island, Korea, November 9-11, 2006, Revised Selected Papers (LNCS) Marcin S. Szczuka, Daniel Howard, Dominik Slezak, Haeng-Kon Kim, Tai-Hoon Kim, Il Seok Ko, Geuk Lee, and Peter M. A. Sloot (Eds.), Vol. 4413. Springer, 42-50. https://doi.org/10.1007/978-3-540-77368-9_5
[5] Andrea Calì. 2004. Query Answering by Rewriting in GLAV Data Integration Systems Under Constraints. In Semantic Web and Databases, Second International Workshop, SWDB 2004, Toronto, Canada, August 29-30, 2004, Revised Selected Papers, Christoph Bussler, Val Tannen, and Irini Fundulaki (Eds.), Vol. 3372. 167-184. https://doi.org/10.1007/978-3-540-31839-2_13

[6] Diego Calvanese, Martin Giese, Dag Hovland, and Martin Rezk. 2015. OntologyBased Integration of Cross-Linked Datasets, See [2], 199-216. https://doi.org/10. 1007/978-3-319-25007-6_12

[7] Carlo Curino, Hyun Jin Moon, Alin Deutsch, and Carlo Zaniolo. 2013. Automating the database schema evolution process. VLDB F. 22, 1 (2013), 73-98. https: //doi.org/10.1007/s00778-012-0302-x

[8] Enrico Daga, Mathieu d'Aquin, Alessandro Adamou, and Enrico Motta. 2016. Addressing exploitability of Smart City data. In IEEE International Smart Cities Conference, ISC2 2016, Trento, Italy, September 12-15, 2016. IEEE, 1-6. https: //doi.org/10.1109/ISC2.2016.7580764

[9] Mathieu d'Aquin, Alessandro Adamou, Enrico Daga, Shuangyan Liu, Keerthi Thomas, and Enrico Motta. 2014. Dealing with Diversity in a Smart-City Datahub. In Proceedings of the Fifth Workshop on Semantics for Smarter Cities, a Workshop at the 13th International Semantic Web Conference (ISWC 2014) (CEUR Workshop Proceedings), Tope Omitola, John G. Breslin, and Payam M. Barnaghi (Eds.), Vol. 1280. CEUR-WS.org, 68-82.

[10] Mirko Michele Dimartino, Andrea Calì, Alexandra Poulovassilis, and Peter T. Wood. 2015. Peer-to-Peer Semantic Integration of Linked Data. In Proceedings of the Workshops of the EDBT/ICDT 2015 foint Conference (EDBT/ICDT), Brussels, Belgium, March 27th, 2015. (CEUR Workshop Proceedings), Peter M. Fischer, Gustavo Alonso, Marcelo Arenas, and Floris Geerts (Eds.), Vol. 1330. CEUR-WS.org, 213-220. http://ceur-ws.org/Vol-1330

[11] Oliver M. Duschka, Michael R. Genesereth, and Alon Y. Levy. 2000. Recursive Query Plans for Data Integration. f. Log. Program. 43, 1 (2000), 49-73. https: //doi.org/10.1016/S0743-1066(99)00025-4

[12] Alon Y. Halevy, Anand Rajaraman, and Joann J. Ordille. 2006. Data Integration: The Teenage Years. In Proceedings of the 32nd International Conference on Very Large Data Bases, Seoul, Korea, September 12-15, 2006, Umeshwar Dayal, KyuYoung Whang, David B. Lomet, Gustavo Alonso, Guy M. Lohman, Martin L. Kersten, Sang Kyun Cha, and Young-Kuk Kim (Eds.). ACM, 9-16. http://dl.acm. org/citation.cfm?id=1182635

[13] Bernadette Hyland, Ghislain Atemezing, and Boris Villazón-Terrazas. 2014. Best Practices for Publishing Linked Data. W3C Working Group Note. W3C. https: //www.w3.org/TR/ld-bp/

[14] Yannis Katsis and Yannis Papakonstantinou. 2018. View-Based Data Integration. In Encyclopedia of Database Systems, Second Edition, Ling Liu and M. Tamer Özsu (Eds.). Springer. https://doi.org/10.1007/978-1-4614-8265-9_1072

[15] Craig A. Knoblock, Pedro A. Szekely, José Luis Ambite, Aman Goel, Shubham Gupta, Kristina Lerman, Maria Muslea, Mohsen Taheriyan, and Parag Mallick. 2012. Semi-automatically Mapping Structured Sources into the Semantic Web. In The Semantic Web: Research and Applications - 9th Extended Semantic Web Conference, ESWC 2012. Proceedings (LNCS), Elena Simperl, Philipp Cimiano, Axel Polleres, Óscar Corcho, and Valentina Presutti (Eds.), Vol. 7295. Springer, 375-390. https://doi.org/10.1007/978-3-642-30284-8_32

[16] Domenico Lembo, José Mora, Riccardo Rosati, Domenico Fabio Savo, and Evgenij Thorstensen. 2015. Mapping Analysis in Ontology-Based Data Access: Algorithms and Complexity, See [2], 217-234. https://doi.org/10.1007/978-3-319-25007-6_13

[17] Maurizio Lenzerini. 2002. Data Integration: A Theoretical Perspective. In Twentyfirst ACM SIGACT-SIGMOD-SIGART Symposium on Principles of Database Systems, June 3-5, Madison, Wisconsin, USA, Lucian Popa, Serge Abiteboul, and Phokion G. Kolaitis (Eds.). ACM, 233-246. https://doi.org/10.1145/543613.543644

[18] Luis Martín, Alberto Anguita, Ana Jiménez, and José Crespo. 2008. Enabling Cross Constraint Satisfaction in RDF-Based Heterogeneous Database Integration. In 20th IEEE International Conference on Tools with Artificial Intelligence (ICTAI 2008), November 3-5, 2008, Dayton, Ohio, USA, Volume 2. IEEE Computer Society, 341-348. https://doi.org/10.1109/ICTAI.2008.67

[19] Norman W. Paton, Klitos Christodoulou, Alvaro A. A. Fernandes, Bijan Parsia, and Cornelia Hedeler. 2012. Pay-as-you-go data integration for linked data: opportunities, challenges and architectures. In Proceedings of the 4th International Workshop on Semantic Web Information Management, SWIM 2012, Scottsdale, AZ, USA, May 20, 2012, Roberto De Virgilio, Fausto Giunchiglia, and Letizia Tanca (Eds.). ACM, 3. https://doi.org/10.1145/2237867.2237870

[20] Anish Das Sarma, Xin Dong, and Alon Y. Halevy. 2008. Bootstrapping pay-asyou-go data integration systems. In ACM SIGMOD International Conference on Management of Data, SIGMOD 2008, Vancouver, BC, Canada, Jason Tsong-Li Wang (Ed.). ACM, 861-874. https://doi.org/10.1145/1376616.1376702

[21] Lukasz Strobin and Adam Niewiadomski. 2016. Integration of Multiple Graph Datasets and Their Linguistic Summaries: An Application to Linked Data. In Artificial Intelligence and Soft Computing - 15th International Conference, ICAISC 2016, Proceedings, Part I (LNCS), Leszek Rutkowski, Marcin Korytkowski, Rafal Scherer, Ryszard Tadeusiewicz, Lotfi A. Zadeh, and Jacek M. Zurada (Eds.), Vol. 9692. Springer, 333-343. https://doi.org/10.1007/978-3-319-39378-0_29 